showed similar associations with testing positive in CSI (OR 2.4 [95\% CI 2.1 to 2.7]) and the STI centres (OR 1.2 [95\% CI 1.0 to 1.3]), but the model basing ethnicity on country of birth of a person and his parents had a better fit (higher likelihood). Self-defined ethnicity may allow for more personal input, this however also makes it a dynamic variable: in the second round of CSI, 15\% of the immigrants identified themselves by a different ethnicity than in the first round see Abstract P1-S4.02 Figure 1.

Conclusions Both self-defined ethnicity and ethnicity based on the country of birth of a person and his parents, can be used to detect young persons at a higher risk of Chlamydia infection. However the definition of ethnicity based on the country of birth explains variation in the Chlamydia data better and is objective and constant, whereas self-defined ethnicity would disregard a large part of the young population at higher risk for Chlamydia infection.

\section{P1-S4.03 USING ORGANISM LOAD OF CHLAMYDIA TRACHOMATIS AND NEISSERIA GONORRHOEAE IN CLINICAL SPECIMENS AS AN EPIDEMIOLOGIC TOOL}

\author{
doi:10.1136/sextrans-2011-050108.147
}

${ }^{1} \mathrm{~B}$ Van Der Pol, ${ }^{2} \mathrm{~A}$ Pantone, ${ }^{3} \mathrm{~J}$ Williams. ${ }^{1}$ School of Public Health, Indiana University, Bloomington, USA; ${ }^{2}$ School of Medicine, Indiana University, Indianapolis, USA; ${ }^{3}$ School of Medicine, Indiana University, Indianapolis, USA

Background The Abbott Realtime m2000 system (m2000) is a qualitative real-time PCR assay for the detection of CT and NG that has the capability to provide a relative measure of target DNA. Results from the m2000 were used to determine CT and NG organism load by comparing the delta cycle (DC) value of each specimen to a set of lab developed standards containing known concentrations of each organism.

Methods Vaginal swabs and male urine specimens were evaluated. Six standards of each organism were prepared by inoculating collection tubes with lab strains in concentrations ranging from 0 to $4 \times 105$ organisms. The $\log 10$ organism load for each positive specimen was determined by comparing the DC value to the calibration curve. Self-reported symptoms were available for each patient.

Results A total of 99 vaginal and 284 urine specimens were available for analysis. There was no statistical difference in DC value of mean organism load by gender. Neither was there a difference based on the presence or absence of symptoms in people infected with CT. For
NG, there was a significant difference in mean $D C$ value and organism load by gender ( $<0.001$ for both DC and organism load) with men having higher loads. In NG positive men the mean DC was 15.2 [95\% CI 14.7 to 15.8] and 11.4 [95\% CI 9.0 to 13.7] for men with and without symptoms $(\mathrm{p}=0.003)$. This translated in mean $\log 10$ organism loads of 6.5 [95\% CI 6.3 to 6.6] and 5.4 [95\% CI 4.7 to 6.1] for men with and without symptoms ( $p=0.005)$. In NG positive women there was no difference in organism load based on presence or absence of symptoms $(p=0.220)$.

Conclusions Advantages to using this methodology include being able to quantify organism load from specimens obtained for routine diagnostic testing, using standardised test reagents that can be purchased commercially, and using an automated platform. Even in those settings that do not have the capacity for calibration, the DC values may provide useful relative loads. This exploratory study demonstrated the feasibility of using this method to obtain relative quantitation measures. Application of this tool to epidemiologic questions using larger data sets may prove useful.

\section{P1-S4.04 BIASES IN THE DESIGN OF STUDIES ASSESSING THE ROLE OF SEXUALLY TRANSMITTED INFECTIONS AS HIV RISK FACTORS}

doi:10.1136/sextrans-2011-050108.148

J O'Hagan, M Lipsitch. ${ }^{1}$ Harvard School of Public Health, Boston, USA

Background For over 20 yearss sexually transmitted infections (STIs) have been studied as risk factors for HIV infection. We show how the design of studies in this field has inherent biases due to inappropriate confounder definition, coinfection, and heterogeneity in exposure to HIV. We use herpes simplex virus 2 (HSV2) to illustrate these biases and show that use of a serodiscordant couple design can remove them. Such findings are timely given the interest in using HPV vaccination for HIV prevention based on similar data.

Methods We developed an individual based model (IBM) using published data from an existing STI IBM (STDSIM). This model permits simulation of multiple cohort studies to show the direction and magnitude of these biases. The model is written in Matlab. Analyses were performed using Cox regression in SAS.

Results We identified four causes of bias. (1) While confounding by sexual behaviour is widely appreciated it is less well understood that perfect measurement of sexual behaviour will not permit adequate control of confounding as data on the frequency of HIV exposure is

Abstract P1-S4.04 Table 1 Comparison of standard study design and serodiscordant couples study design for estimating the per-sex act risk of HIV infection among HSV2 infected individuals compared to HSV2 uninfected individuals

\begin{tabular}{llll}
\hline & $\begin{array}{l}\text { Null Median HR } \\
\text { (Lowest HR, Highest HR) }\end{array}$ & $\begin{array}{l}\text { Different transmissibility } \\
\text { Median HR (Lowest HR, } \\
\text { Highest HR) }\end{array}$ & $\begin{array}{l}\text { Coinfection increases } \\
\text { hiv infectiousness Median } \\
\text { HR (Lowest HR, Highest HR) }\end{array}$ \\
\hline $\begin{array}{lll}\text { Expected } \\
\text { Standard Design }\end{array}$ & 1 & 1 & $\begin{array}{l}\text { Susceptibility Median HR } \\
\text { (Lowest HR, Highest HR) }\end{array}$ \\
Serodiscordant Couples & $1.32(0.84,2.26)$ & $1.58(0.64,1.44)$ & $1.26(1.01,1.56)$ \\
10 & $1.07(0.70,2.11)$ & $1.03(0.72,1.25)$
\end{tabular}

20 simulations were performed for each bias scenario. Simulations were started in 1930. Cohort studies were conducted from 1992-95, which is the period during which the data used to parameterise the model were collected in rural Tanzania. The data from each run was analysed using Cox proportional hazards regression in two ways, both of which enrolled sexually active index subjects who were HIV negative and who could be HSV2 positive or negative:

1) Standard design - data on exposure to infection was not incorporated into the analysis.

2) Serodiscordant couples design - only those individuals who had an HIV infected partner were included and only for the length of time they were in the partnership.

All analyses adjusted for age in 5-year intervals, gender, and the baseline number of lifetime partners. Results presented are the median, lowest, and highest point estimates for the HR calculated from these runs. HIV and HSV2 per-sex act transmission probabilities were both equal to 0.02 unless otherwise stated.

Null: No interaction between HSV2 and HIV.

Different Transmissibility: No interaction between HSV2 and HIV. HSV2 per-sex act transmission probability $=0.03$.

Coinfection Increases HIV Infectiousness: Coinfected individuals have twice the HIV per-sex act transmission probability than individuals infected with HIV alone (ie, 0.04 vs 0.02 respectively). Susceptibility: The HIV per-sex act transmission probability to an HSV2 infected individual is 10 times higher than to an HSV2 uninfected individual (ie, 0.2 vs 0.02 respectively).

Note: Susceptibility is not affected in the first three scenarios. Also, parameter values have been changed from disease specific values to permit each bias to be presented separately. Concurrent partnerships are not permitted. Full sensitivity analysis results which relax these restrictions are not shown. 